\title{
From risk to function
}

\section{In association with the Wellcome Trust, we are pleased to announce the second Genomics of Common Diseases conference to be held September 6-9, 2008, in Cambridge, MA, USA.}

$\mathrm{R}$ eviewing last year's meeting, Enrico Petretto and colleagues (Nat. Genet. 39, 1299-1301; 2007) emphasized that recent successes in complex disease genetics rest upon rapid technological advances and the excellent communication and collaboration forged in the genome sequencing community and the SNP Consortium. They also stressed the importance of studying gene networks rather than single genes in the pathogenesis of disease. Finally, they noted that an evolutionary as well as a physiological perspective is required to understand the startlingly diverse genetic architectures of common diseases.

What to expect from this year's meeting (http://www.nature. com/natureconferences/gcd2008/index.html)? Over the past year, many new sequencing tools have been added for strategically resequencing candidate genes for rare and common variants alike. Bioinformatic tools have been launched for sequence assembly and comparison. Statisticians have developed methods for marker imputation and for rigorous association studies with structural variants.

With these new tools, more difficult targets have been tackled. Sequencing clonal populations of cancer cells has revealed clues into how genomes diversify in disease, and we now have hints of the causal contributions of mutation and rearrangement (Science 314, 268-274; 2006; Nat. Genet. 40, 722-729; 2008). Perhaps similar somatic sequencing methods can soon be used to detect mosaic somatic changes during aging.

As a community, we have realized that there are more layers to complex traits than genes and environment, but we are still struggling to connect the meshed network of chromatin and DNA modification that lies between the genotype and even gene expression phenotypes. The desperately overworked term 'epigenetics' covers all these processes. Together with the connections between genetic elements that is better termed the 'network wiring diagram', DNA and chromatin modification are likely to hold the key to understanding how genetic variation is tolerated and buffered, and whether variants exert strong or weak effects on phenotypic traits.

The success of the common variant association method enabled by the HapMap rolls on from hit to hit, and as the sizes of the studies grow, so more replicated associations with new loci emerge. Meta-analysis by Eleftheria Zeggini and colleagues (Nat. Genet. 40, 638-645; 2008) revealed a total of 16 loci associated with type 2 diabetes, and Jeffrey Barrett and colleagues ( $\mathrm{p} 955$ ) now report 32 loci associated with Crohn's disease. Common variants predisposing to multiple diseases have now been found in several studies, sometimes conferring opposite risks of each disease.

Although common variants typically have small effects on absolute risks of disease, there is already evidence that the new research findings can be usefully applied. Combining recently discovered common variants at seven loci associated with breast cancer in a multiplicative model explaining just 5\% of the genetic risk, Pharoah et al. (New. Engl. J. Med. 358, 2796-2803; 2008) calculate that, by age $41,5 \%$ of women in the UK population will have the same 10 -year risk of breast cancer $(2.3 \%)$ as an average woman of 50, the age threshold at which annual mammographic screening is now offered.

But how will people really use personal genomic information? Colleen McBride and colleagues (p 939) are putting multi-locus genotypes and associated risk information in the hands of individuals, in an attempt to dissect the mechanisms of motivation and behavioral modification.

In the era of personal genomics, with next-generation sequencing of thousands of human genomes just beginning, the focus of common disease genetics is already shifting from identification of loci and risks. We anticipate an intensification of functional genomics, investigation of the consequences of variation on gene expression and the effect of variation on the functioning of pathways and systems. 